\title{
Research Paper: \\ The Strategies Used by Visually Impaired and Blind People in Iran to Cope With Difficulty Performing Daily Living Activities
}

\author{
Faezeh Aghazadeh ${ }^{1}(\mathbb{0}){ }^{*}$ Abbas Riazi ${ }^{2}$ (i), Mohammad Kamali ${ }^{3}$ (i)
}

1. Department of Optometry, School of Rehabilitation, Iran University of Medical Sciences, Tehran, Iran.

2. Vision Research Center, Iran University of Medical Sciences, Tehran, Iran.

3. Department of Health Education \& Promotion, School of Health Management and Information Sciences, Iran University of Medical Sciences, Tehran, Iran.

\begin{tabular}{|c|c|}
\hline $\begin{array}{l}\text { Use vour device to scan } \\
\text { and read the article online }\end{array}$ & iazi A Kamali M TThe Strateoies Used by Visually Imnaired and Blind Peonle in Iran to Cone \\
\hline 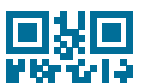 & $\begin{array}{l}\text { With Difficulty Performing Daily Living Activities (Persian)]. Archives of Rehabilitation. 2021; 22(2):196-209. https://doi. } \\
\text { org/10.32598/RJ.22.2.3243.1 }\end{array}$ \\
\hline apt & dol'https://doi.org/10.32598/RJ.22.2.3243.1 \\
\hline
\end{tabular}

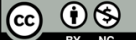

Received: 22 Aug 2020 Accepted: 22 Nov 2020 Available Online: $01 \mathrm{Jul} 2021$

Keywords:

Coping strategies, Quality of life, Visual impairment, Low vision, Blindness

\begin{abstract}
Objective This study was conducted to investigate the strategies used by visually impaired and blind people in Iran to cope with difficulty performing daily living activities.

Materials \& Methods This research is a qualitative study using a content analysis method. The participants were 18 visually impaired and blind people who were purposefully selected from those referred to the Visual Aid Research Center of Iran University of Medical Sciences in Tehran City, Iran. A semistructured interview was first used using general questions about some daily living activities to discover the participants' experiences. Attempts were made to allow participants to express their experiences independently without the help of the interviewer. The interviews were recorded as an audio file. After the interview, the audio files were transcribed and analyzed using thematic content analysis. The key sentences were extracted from the main texts, and the strategies or concepts extracted from those sentences were identified. Common strategies were put under one category. After the data saturation, when no new concept was found in the sentences, the interviews ended. By examining similar strategies in a category, subcategories were defined, and finally, similar subcategories were placed in a more general category, and the main themes or strategies were identified.

Results The Mean $\pm S D$ age of the participants was $39.5 \pm 11$ years, ranging from 22 to 66 years. Of 18 participants, 7 were blind, 7 had severe visual impairment, and 4 had moderate visual impairment. Their duration of blindness or visual impairment was more than five years. In terms of education, 7 had no high school diploma, and 11 had a high school diploma or higher degree. Vision loss was congenital in some subjects and acquired in others. We extracted 8 main themes or strategies used by the subjects to cope with difficulty performing daily living activities. These themes are trust in others, use of alternative senses, efficient vision care, use of technology, optimization of the living environment, avoiding a specific activity, increasing emotional intelligence, and use of intelligence and memory.

Conclusion The strategies used by the visually impaired and blind people in Iran are entirely personal and innovative and play an influential role in increasing their quality of life. According to their own statements, these strategies can solve their many problems in performing daily living activities.
\end{abstract}

${ }^{*}$ Corresponding Author:

Abbas Riazi, PhD.

Address: Vision Research Center, Iran University of Medical Sciences, Tehran, Iran.

Tel: +98 (21) 22222059

E-Mail: abbas.riazi@gmail.com 


\section{Extended Abstract}

\section{Introduction}

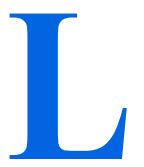

ow vision is a visual impairment. In this condition, a person's vision does not improve with any medication, surgery, and glasses, and contact lenses. Due to population growth in different parts of the world, visual impairment and blindness are increasing in the world [1]. The causes of low vision and blindness are different and can occur in any race, ethnicity, nationality, and family history [2]. According to many studies, vision loss declines the quality of life [3]. Vision is a crucial factor in mental health. A person with visual impairment has difficulty doing their daily chores and sometimes becomes dependent on others for performing simple tasks. This dependence can lower the self-esteem of a visually impaired person and cause dissatisfaction with life and depression [4]. Many solutions have been suggested to improve the quality of life of these people. One of these strategies is applying practical strategies that are invented and used by blind or visually impaired people to do their daily living activities [5]. Also, therapists and rehabilitation specialists should pay attention and be aware of these strategies and encourage visually impaired people to use them [6].

Therapists who assess visual acuity should consider their patients' coping mechanisms and refer them for positive counseling and training in appropriate ways [3]. One study examined the psychological impact of vision impairment and coping strategies in retired working women and how to cope with their low vision. Women could apply different coping strategies in their daily work, such as having a positive attitude, re-learning skills, and using visual aids [7]. Avoidance, problem-solving, and active search for social support can be other strategies [5]. One study examined coping with vision-related goal interference in midlife. The researchers found that middle-aged adults with vision impairment use the following coping strategies: internal resource use, new approach use, technology use, help use, and psychological self-regulation [8]. Hope, coping, and functional ability are other strategies reported in another study [9]. Products and technology, physical environment, support and relationships, attitudes, and services and policies were environmental factors affecting the performance of blind people in daily living activities. This study aims to investigate the individual applied coping strategies among visually impaired and blind people in Iran.

\section{Materials and Methods}

This research is a qualitative study using content analysis. The samples were purposefully selected from people with visual impairments living in Tehran City referred to the Visual Aid Research Center of the Faculty of Rehabilitation, Iran University of Medical Sciences. First, the participant's ability to take part in the research was assessed by performing a perception test in the form of asking a series of simple questions. The purpose of the study was explained to them or their families or companions, and their consent was obtained to record their voices during the interview. After preparing a separate record file for each of them, they were assured that their information would remain confidential. A semi-structured interview was conducted using general questions about some daily living activities to discover the experiences of visually impaired and blind people. Attempts were made to allow participants to express their experiences independently without the help of the interviewer. The initial optometric examinations included surveying the history of the disease and treatments performed so far, measuring vision with and without optical correction at near and far distances, assessing the refractive errors, and recording the history of visual aids use. The visual chart used in this study at near and far distances was the standard logarithmic vision chart. Refractive errors were measured by retinoscope. After the initial examinations, the participants were selected and interviewed. The inclusion criteria were having moderate to severe visual impairment or blindness according to the World Health Organization's criteria for the classification of low vision, being over 20 years old with at least five years of visual impairment, being able and willing to participate in the research, and lacking perceptual problems.

The interviews were first recorded. Then, the audio files were transcribed and analyzed based on the thematic content analysis. The key sentences were extracted from the main texts, and the strategies or concepts extracted from those sentences were identified. Common strategies were put in one category. After the data saturation, that is, when no new concept was found in the sentences, the interviews were ended. By examining similar strategies in a category, subcategories were defined, and finally, similar subcategories were placed in more general categories, and the main themes or strategies were identified.

\section{Results}

The participants were 18 blind or visually impaired adults ( 9 females and 9 males) with an average age of 39.5 years. Seven were blind, 7 had severe visual impairment, and 4 had moderate visual impairment with an impairment duration of more than 5 years. In terms of education, 7 had no 
high school diploma, and 11 had a high school diploma or higher degree. Vision loss was congenital in some participants and acquired in others. Congenital glaucoma, premature retinopathy, optic nerve atrophy, trauma, night blindness, trachoma, and diabetic retinopathy were the causes of visual impairment of the participants.

A total of 676 key sentences were extracted from the interviews. Of them, 212 concepts or applied strategies were obtained. By categorizing common strategies, 35 sub-strategies and finally, 8 main strategies or themes were obtained, which are presented as follows:

Trusting others: All participants were reported that they trust others in performing their daily living activities. "When using an ATM, I tell someone to enter the password and see how much money I've received".

Use of alternate senses: All participants used other senses to replace the sense of vision. "At the time of study at university, I gave the pamphlets to someone to read, and I recorded his voice" (sense of hearing). "I touch the excess hair on my face with my finger, then I detect its place and remove it with a tweezer. I use my hands a lot" (sense of touch); "If the food smells, I'll know what the food is, like celery. But if it does not smell, I'll not be able to recognize it" (sense of smell). "I know from the taste that it is, for example, turmeric or pepper! If there is something I can't recognize, I'll taste a little of it" (sense of taste). "It's a little difficult for us, for example, since the bathroom floor is slippery, we always walk or sit in the bathroom carefully so that we do not slip, because we can't see the front of us" (balance). "While making a cutlet, I slide a spoon under the meat. When it makes a hissing sound, I know it's roasted. Then, I turn it onto its other side" (proprioception).

Use of technology: Most participants used science-based tools and techniques. "I mostly use white canes outdoors. It's not applicable indoors" (advanced equipment). "If I want to go somewhere by taxi that can be ordered online, I specify the destination early on my phone, or I ask others to call" (modern lifestyle).

Optimization of living environment: Many participants became more adaptable to their environment by optimizing their surroundings. "To the relatives who sometimes wash the dishes in our house, I tell them to put everything in its place and do not change their places" (no displacement). "Well, the toothbrushes we once bought were almost identical such that I couldn't differentiate them. So, I put a money rubber band around my toothbrush" (marking). "In the bathroom, I have a closet like a plastic cupboard that is personal drilled into the wall. My shampoo, towel, and other stuff are there. It's just for me, and no one is allowed to touch them" (Separation and classification).

Avoiding a specific activity: "I don't walk on the yellow line because my foot hurts". "I like swimming, but I don't do it; I'm scared to get lost in the water". "I don't like white cane, and it makes me feel bad" (Lack of motivation and having unpleasant experiences).

Increasing emotional intelligence: "Since the age of 9, I accepted that I had an illness that might not have a cure. I mean, it's OK for me. This thought helped me to cope with it and live, to go to university and get a Master's degree" (Accepting disability and positive thinking). "When I brush my teeth, when I comb my hair, when I'm going to get dressed, I look at myself in the mirror spuriously. I may not see, but it is really important for sighted people to look in the mirror. If I don't, it makes me feel bad; I do it because of them, and because I want to be like them" (being like normal people). "Perhaps the biggest change I made in myself was my behavior. I admitted to myself that when I am in a painful situation. I should laugh and rely on myself and not grieve; I have to be able to do my own works" (selfconfidence and self-esteem).

Use of intelligence and memory: "If I want to go to an address, I have to go a few more times to learn” (repetition). "Playing music is much harder for a blind person than for a sighted person because the sighted person looks at the note and plays the instrument. The blind should memorize the note, sentence by sentence, and then play the instrument" (memorizing). "To climb up the stairs, I walk up mostly on the left, which has a fence, because at the right side may be a flower pot". (a logical connection with affairs). "When I go shopping for clothes, someone says it's pale blue; I imagine what it'll be like, then I buy it if I liked it. When I come home, I try to match it with my pants' color; I say, for example, this Manto (a coat wearing by Iranian women) matches with these pants. I then put a blue scarf on it" (Imagination).

Efficient vision use: A small number of participants continued to use their vision despite severe visual impairment. "I pour the tea into a colorless glass. It's hard to see in a porcelain cup. I hold it in front of the light to find out if it is pale or not. I have to see the other side of the tea from one side. Based on my experience, I know how it should be to be good". 


\section{Discussion and Conclusion}

Visually impaired people cannot do things that ordinary people can easily do using their vision [11]. People with visual impairments lose their ability to do certain activities, partially or completely [12]. One of the strategies used by the participants in this study was to trust others in performing activities. The type of trust depends on factors such as visual impairment, personal mood, family conditions, society, and the type of activity [13]. Blind people use other senses to compensate for their vision loss. This ability is due to the reorganization of the vision-related areas of the brain to process non-visual information such as sound and tactile stimuli [10]. Another strategy was the use of technology. The blind and visually impaired people use much technology-based equipment for entertainment, education, business, communication, and so on [14]. An essential tool for mobility and orientation used by these people is the white cane [15]. Advances in technology can help blind people be more mobile in the environment. A system called "smart vision" has been proposed for blind people, which allows them to move around in unfamiliar environments using GPS [16]. Moreover, a shoe has been designed that can be used to detect obstacles around a blind person while walking. The mechanism of informing is vibration in the ankle area [17]. Nevertheless, Iranian blind and visually impaired people use old canes.

Some participants in this study optimized their living environment to adapt to vision problems. Marking was also reported to be used to identify people and things, to find their way around, or even to find the clothes they want. Another strategy was using emotional intelligence. Emotional intelligence can use four skills: self-awareness, self-management, social awareness, and relationship management. The first two skills are related to how the person can understand and control their emotions. The other two skills are related to the person's relationship with others by which s/ he can understand the emotions of others and manage them in relation to herself/himself [18]. One of the factors that increased emotional intelligence in several participants in this study was communication with other visually impaired and blind people. In addition to increasing self-confidence, this communication informed the person about new strategies used by others. Mental health is one of the most important issues that seem to need more research [18]. According to many psychologists, intelligence is a set of talents with which we recognize, remember, and apply the elements of culture to solve everyday problems and adapt, fix, or change the environments [18]. One participant stated that if he climbs up the stairs on the side where there is a fence, he probably will not hit anything because most of the time, the flower pots are placed near the wall. Another partici- pant stated that if he crosses the street with the crowd, the probability of an accident will decrease because the driver can see the crowd and slow down. Some participants used imagination to recognize objects and their surroundings and to understand colors [18].

An effective vision is used when a person's daily life is affected by vision loss [19]. The goal of effective vision enhancement is not to increase vision but to use vision more effectively [19]. The purpose of vision rehabilitation for those with visual impairment is to train independent people who are capable of having a job or business and being able to enjoy their lives. It is a type of modern rehabilitation for the visually impaired and includes assessing remained efficient vision, providing visual rehabilitation therapies, etc. [13]. Strategies used as visual rehabilitation by participants in this study included the use of contrast, ambient light control, visual aids, and various optical or electronic magnifiers such as a magnifying glass, color vision assistance, and other similar techniques. Among the participants, some people lacked any separate optical correction for nearby objects and did not know that they had presbyopia. They had not seen a therapist (optometrist or ophthalmologist) for years.

\section{Study recommendations}

In the present study, the cause of visual impairment and blindness was not crucial in the purposeful selection of the samples. It is suggested that in a similar study, the type of disease be used as a criterion for selection to examine the differences between visually impaired and blind people with different causes of blindness in choosing a coping strategy. Moreover, since the interview questions were limited to predetermined topics and contexts, in other studies, different contexts can be included in the questions to examine other aspects of the life of the visually impaired and blind people.

Blind and visually impaired Iranian people use unique coping strategies that can effectively improve the quality of life of these people. Factors such as the patient's mood, family perception of the disability, society's general culture in recognizing and communicating with the patients, decisions, and programs of medical centers and support organizations can play an essential role in using these strategies by blind and visually impaired people. Factors such as being rejected by the family after suffering from blindness, especially the spouse or obsessive affection and extreme worries from family members, can affect the independence of the blind or visually impaired people and prevent the use of practical coping strategies by them and thus reduce their quality of life. Given that the present study was conducted qualitatively, and the interviews with the participants were based on questions with predetermined contexts, the report- 
ed strategies cannot cover all aspects of the life of the visually impaired or blind people in Iran and cannot be generalized to all visually impaired or blind people. We hope these results be considered a solution for other visually impaired and blind people to do their daily activities and increase their quality of life.

\section{Ethical Considerations}

Compliance with ethical guidelines

All ethical principles are considered in this article. The participants were informed about the purpose of the research and its implementation stages. They were also assured about the confidentiality of their information and were free to leave the study whenever they wished, and if desired, the research results would be available to them.

Funding

This research did not receive any grant from funding agencies in the public, commercial, or non-profit sectors.

Authors' contributions

All authors equally contributed to preparing this article.

Conflict of interest

The authors declared no conflict of interest.

\section{Acknowledgments}

Thanks to the Khazaneh Center for the Visually Impaired and Blind and all the dear and visually impaired loved ones who cooperated with us in conducting this research. 


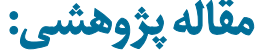 \\ استراتثى هاي منحصربهفرد ابداعى توسط كمبينايان ونابينايان ايرانى در انجام فعاليتهاي روزمرهزندًي}

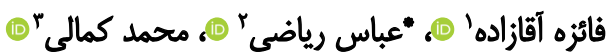

ا. ا. كروه بينايى سنجى، دانشكده توانبخشى، دانشكاه علوم يزشكى ايران، تهران، ايران.

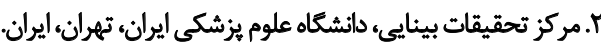

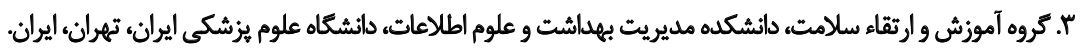

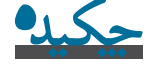

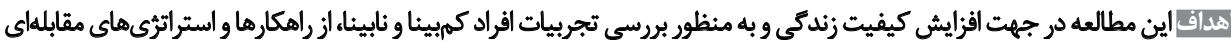

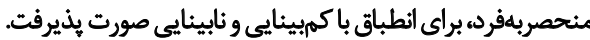

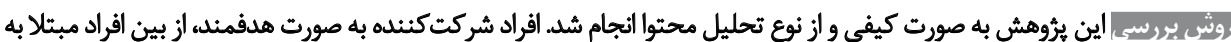

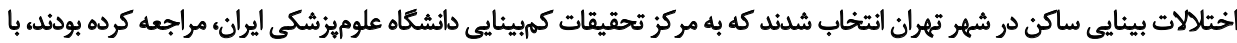

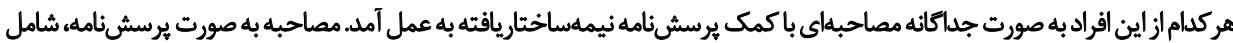

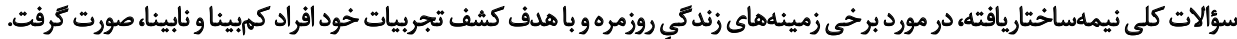

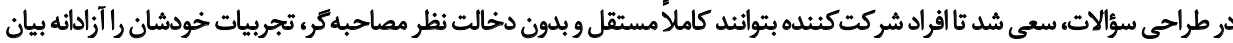

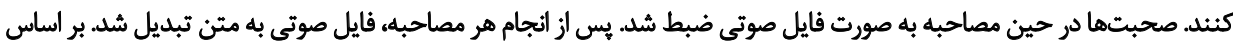

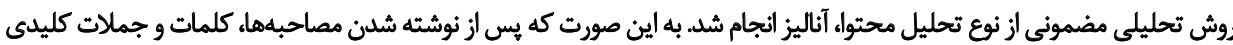

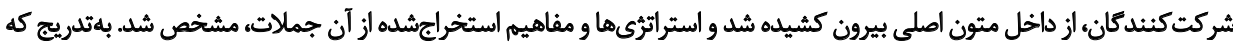

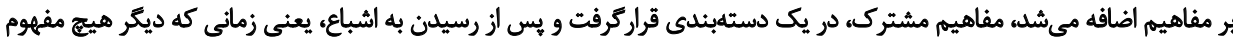

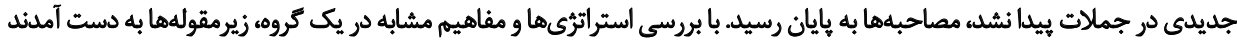

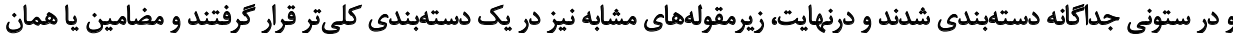

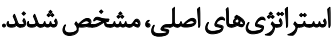

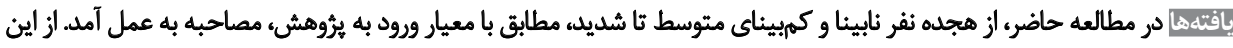

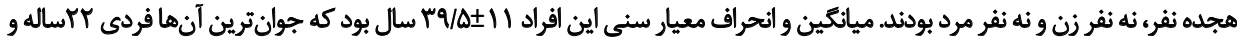

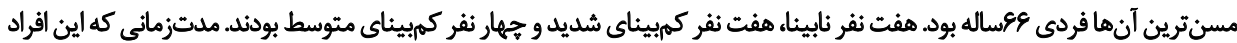

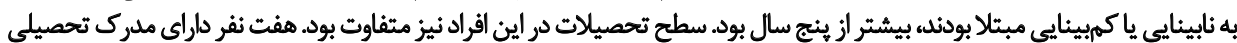

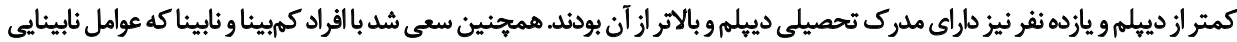

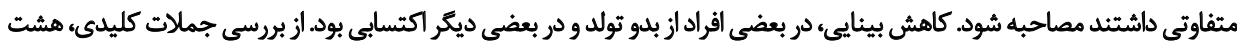

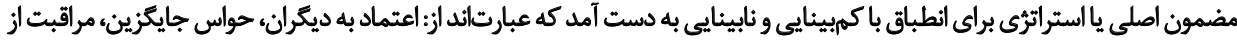

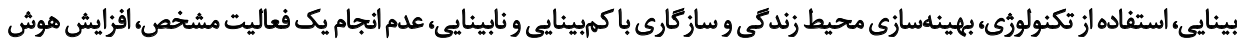
هيجاني و بهكاركيرى هوش و حافظه.

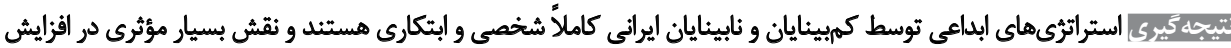

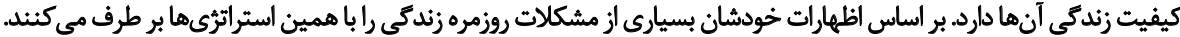

تاريخ دريافت: ا• شهريور 99\%1

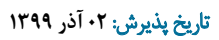

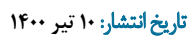

كليدواروهاء:

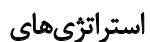
مقابلهاي، ريفيت زندكى، اختلالات بينايى، كمبينايیى، ثابينايى 
هدف از مطالعه حاضر، بررسى استراترىهاى كاربردى شخصى و ابداعى در بين كمبينايان و نابينايان ايرانى بود.

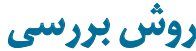

مطالعه به صورت كيفى و از نوع تحليل محتوا انجام شد.

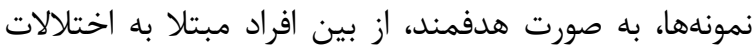

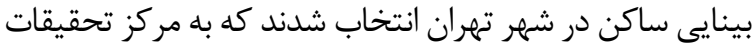

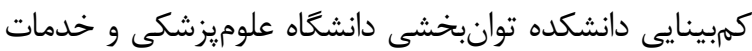
بهداشتىدرمانى ايران مراجعه كردند.

در مطالعه حاضر، از هجده نفر نابينا و كمبيناى متوسط تا

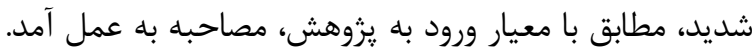

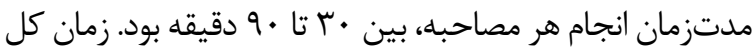

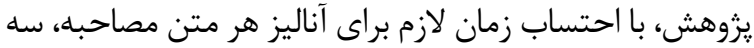

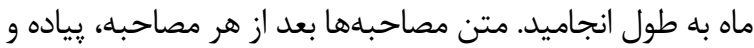

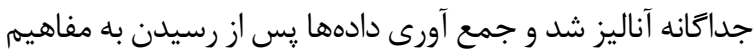

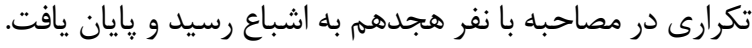

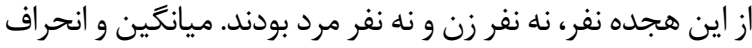

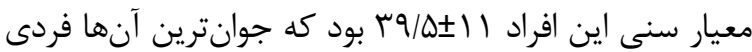

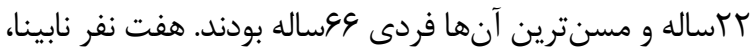

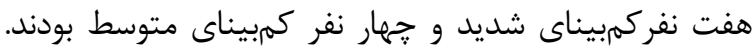

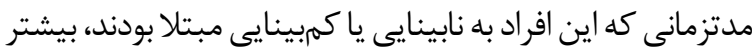

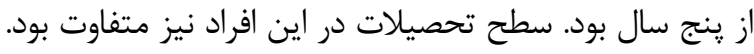

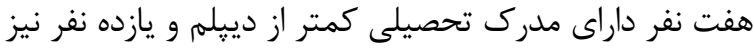

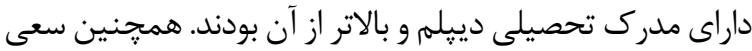

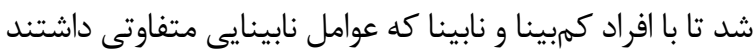

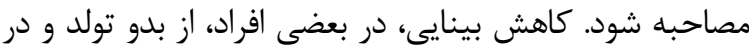

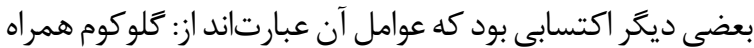

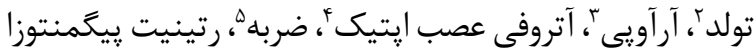

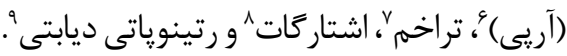

در ابتداى هر مصاحبه، با انجام تست ادراك كه در قالب ترسيدن

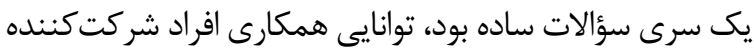

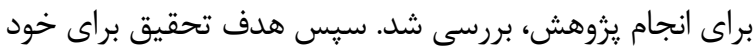

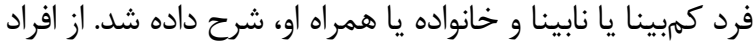

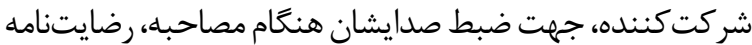

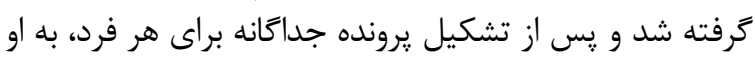

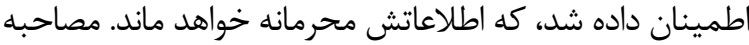

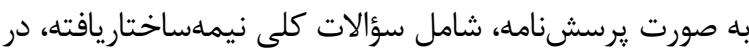

2. Congenital glaucoma

3. Retinopathy of Prematurity (ROP)

4. Optic atrophy

5. Trauma

6. Retinitis Pigmentisa

7. Trachoma

8. Stargardt

9. Diabetic retinopathy مelat

كمبينايى'، به معنى اختلال بينايى است. اين شرايط به كَونهاى

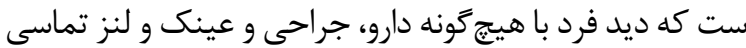

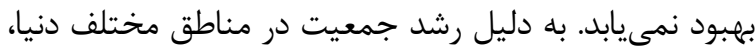

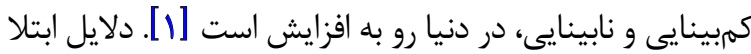

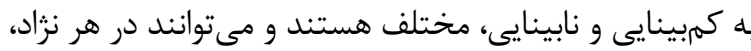

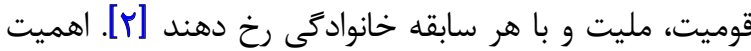

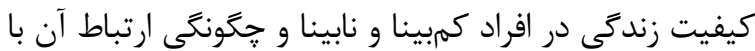

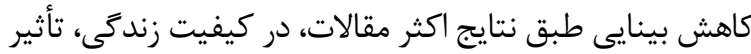

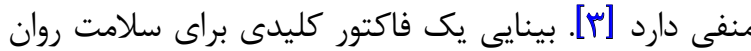

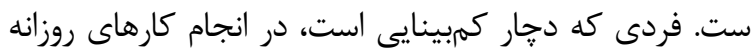

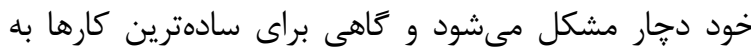

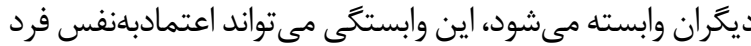

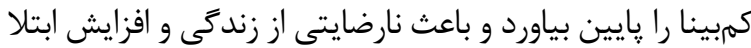

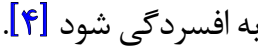

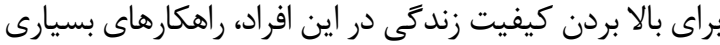

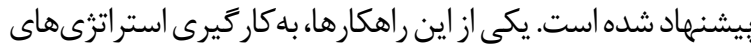

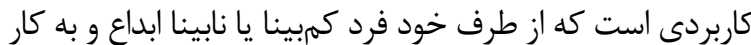

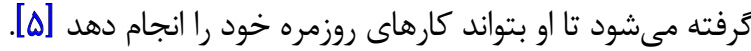

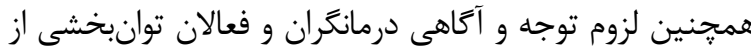

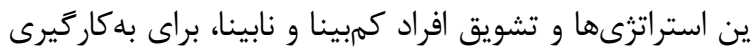

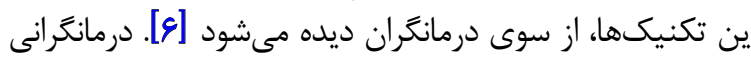

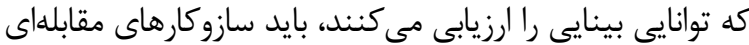

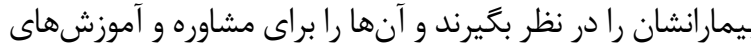

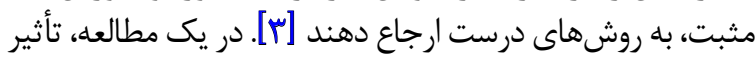

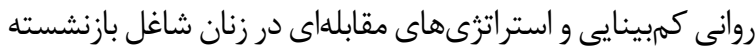

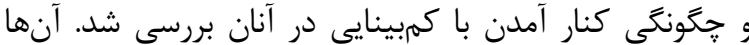

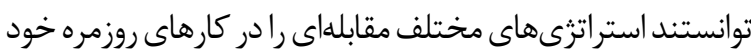

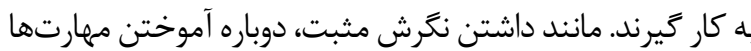

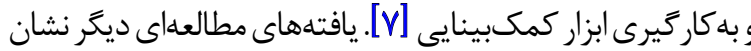

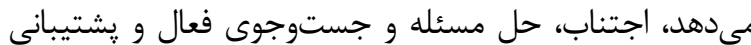

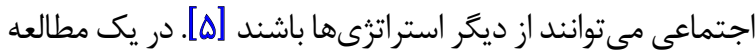

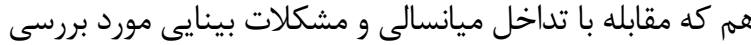

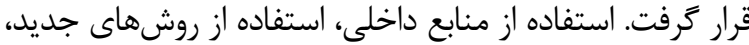

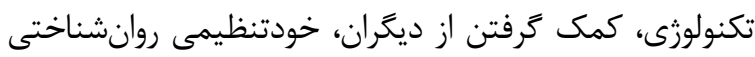

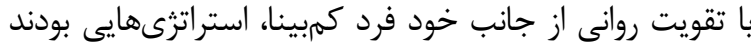

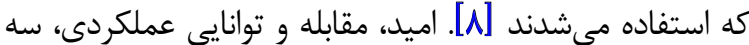

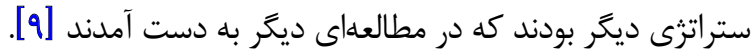

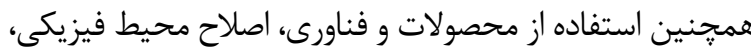

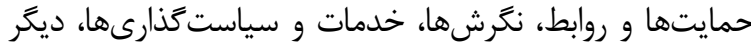

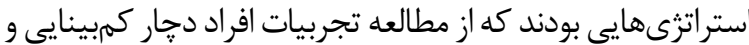

نابينايى اكتسابى به دست آمدند [ـ [1]

1. Visual Impairment 


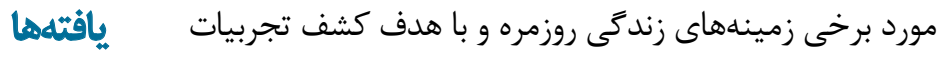

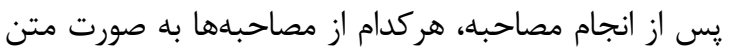

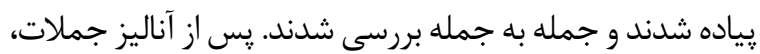

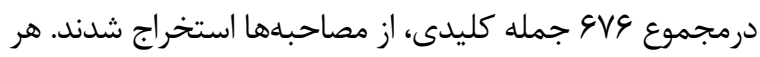

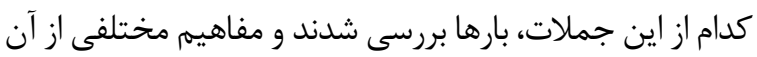

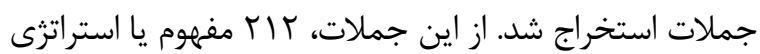

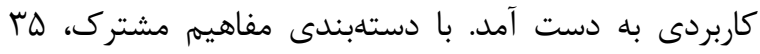

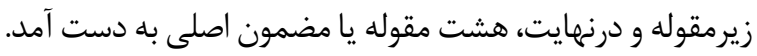

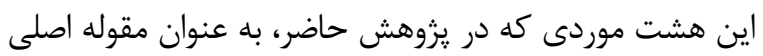

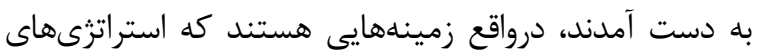

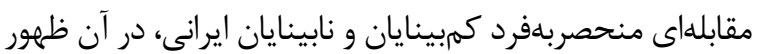

$$
\begin{aligned}
& \text { קيدا كرده است و عبارتاند از: } \\
& \text { 1. اعتماد به ديخران؛ } \\
& \text { r. حواس جايكزين؛ } \\
& \text { r. استفاده از تكنولوزى؛ } \\
& \text { f. بهيناهازى محيط زندگى } \\
& \text { ه. اجتناب از انجام يك فعاليت مشخص؛ } \\
& \text { 9. افزايش هوش هيجانى؛ } \\
& \text { V. به كارگيرى هوش و حافظه؛ } \\
& \text { 1. مراقبت از بينايى كارآمد. }
\end{aligned}
$$

اعتماد به ديمكران

همه شركتكنندًان، در انجام كارهاى خود، به نوعى به ديخران اعتماد مى كردند.

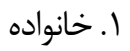

"براى تشخيص داروها، از خانواده كمك مى گيرم."

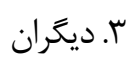

"اهنكام كار باعابر بانك مى گمثم كسى بياد رمز رو برام بزنه، ببينه جقدر يول ريختن برام."

حواس جايكَّنين

همه شركتكنندگان از حواس ديخر براى جايكزينى حس بينايى استفاده مى كردند.

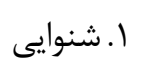

"ادر دانشخاه جزوهها رو مىدادم يكى مى خوند و صداش رو

$$
\text { ضبط مى كردم.《 }
$$

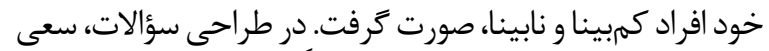

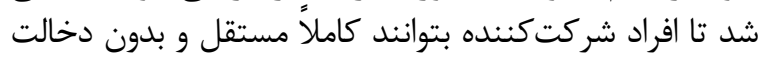

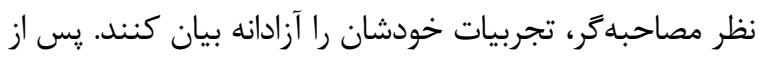

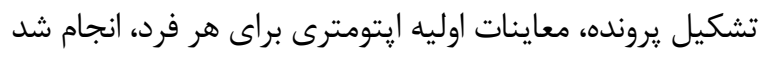

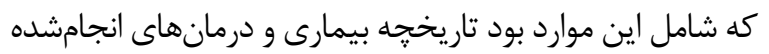

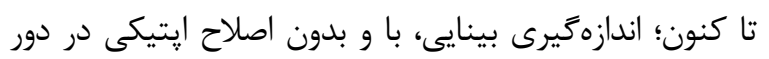

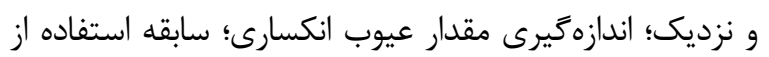
تجهيزات كمكى بينايى.

جارت بينايى استفادهشه در اين مطالعه در فواصل دور و

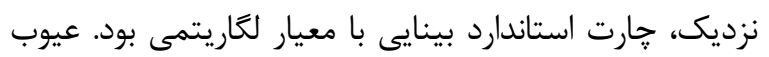

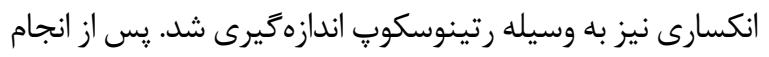

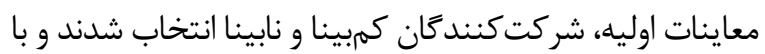

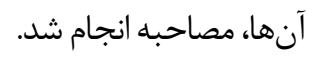
هعيار هاي ورود بل يثروهش • نوع خاصى از بيمارى جشمى و علت كمبينايى و نابينايى در

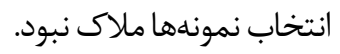
• ملاكى انتخاب، افراد داراى كمبينايى متوسط تا شديد و يا

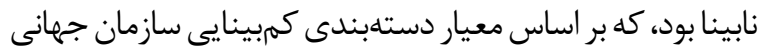

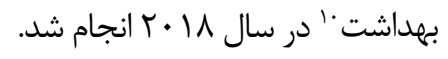

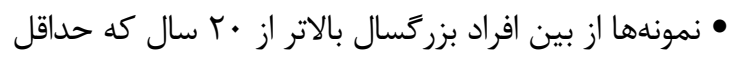

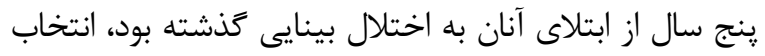
شند.

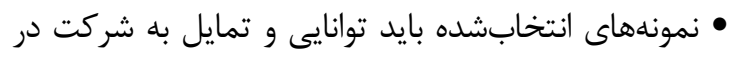

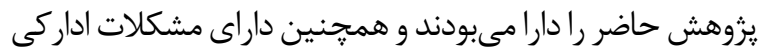

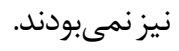
صحبتها در حين مصاحبه به صورت فايل صوتى ضبط شد شد.

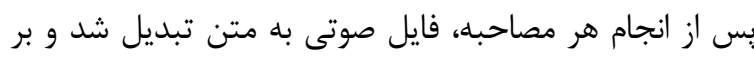

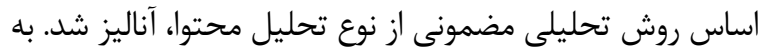

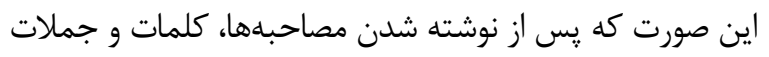

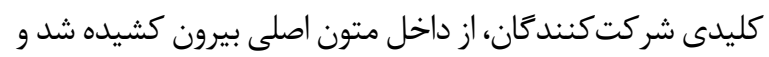

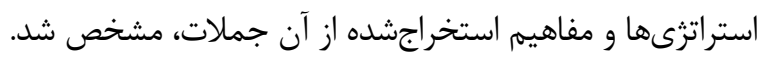

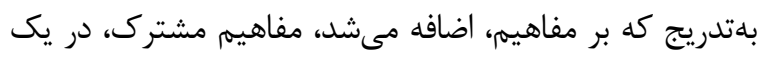

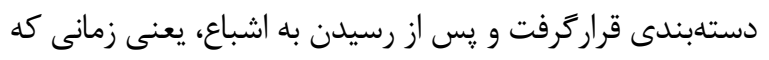

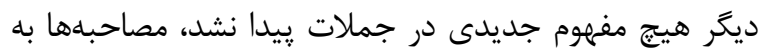

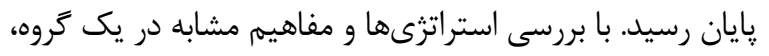

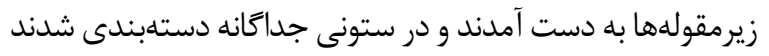

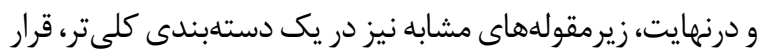
كرفتند و مضامين يا همان استراترىهاى اصلى، مشخص من شدند. 


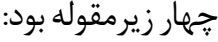

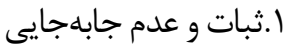

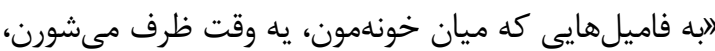
مى گم همه جيرو بذاريد سر جاش، عوض نكنيد.

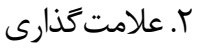

الالهه هميشه بايين شلوارش رو كوتاه مى كنه. من نمى كنهم.

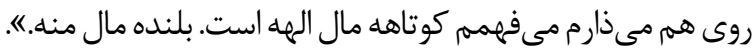
اخب مسواكهايى كه خريديم تقربياً شبيه به هم هستن.

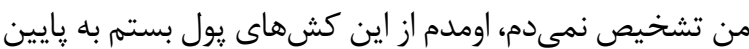

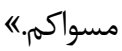

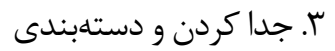

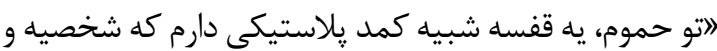

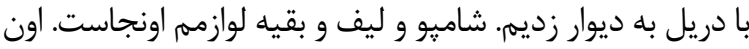
فقط براى منه و كسى بهش دست نمىزنه. اجتناب أز أجام يك فعاليث مشخص يكى از استراترىها براى كمبينايان و نابينايان ايرانى، عدم انجام

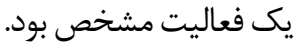
״روى خط زرد راه نمىرم، جون پام درد ميخيره.《

"اتوى كوجه خودمون ترجيح مى دم از ييادهرو نرم، از خيابون

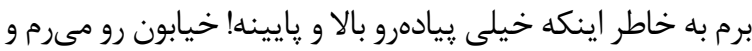
مطمئنم كه جز ماشين خطر ديگداى نيست. "شنا دوست دارم، اما انجام نمى دم، گمم مىشم تو آب." r. عدم داشتن انخيزه و تجربيات ناخوشايند "اعصاى سفيد رو زياد دوست ندارم، حس بدى مى ده بهه." التوبوس زياد سوار نمىشم. خاطره بدى دارم. جيب آدم رو

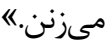
اقزايش هوش هيبانى از جمله استراترىهاى به كار كرفتهشده توسط شركت كنند

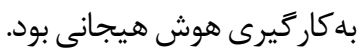
1. يذيرش ناتوانى و انكيزه مثبت خود فرد

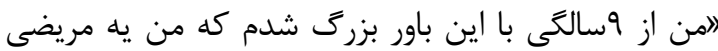

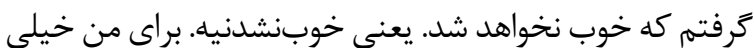

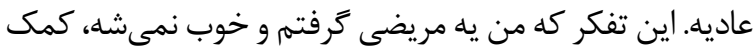

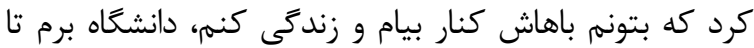

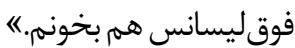

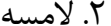

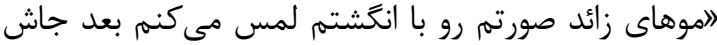

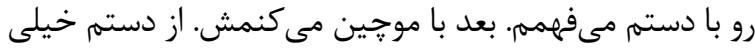

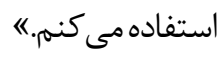

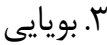
ااكه غذا از بوش معلوم باشه مىفهممم جه غذايى هست، مثلا كرفس، ولى احه بو نداشته باشه نمى بهمهمه."

$$
\text { ( ) جشايى }
$$

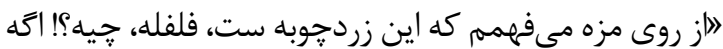

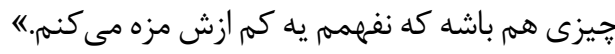

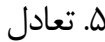

"ابراى جشماى ما يه كم سخته، مثلاً يكى مثل ماها، تون تو

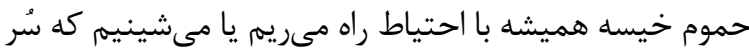

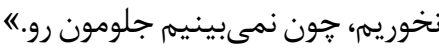

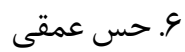

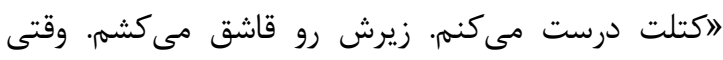

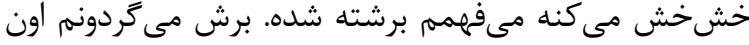

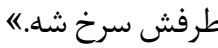

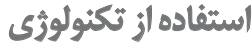
اكثر شركتكنندًان، از ابزارها و تكنيكهاى علممحورو، كه در ارتباط با تكنولوزى هستند، استفاده مى كردند كه شامل ئن ينج

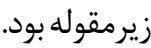

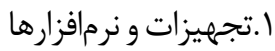
"اتزديك تلويزيون مىشينم، سايزش بزر كه، خوبه.《 (آى اسييك رو گوشيم نصبه. برنامه گَوياى فارسيه. بعد قشنگ

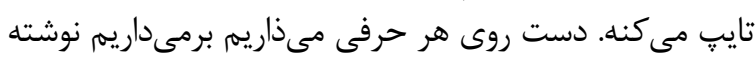

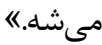
ااز عصاى سفيد بيشتر خارج منزل استفاده مىكنهم. داخل

$$
\begin{aligned}
& \text { منزل كاربردى نداره.ه } \\
& \text { r. سبك زندگى مدرن }
\end{aligned}
$$

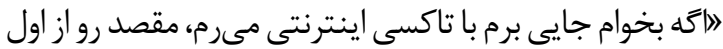

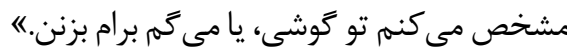

$$
\text { بيئهسازى محيط زندتى }
$$

عده زيادى از شركت كنندكان، با بهينه كردن محيط اطراف

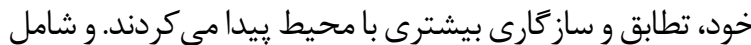




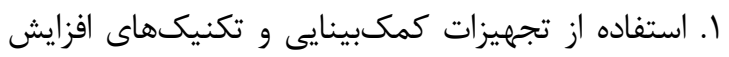

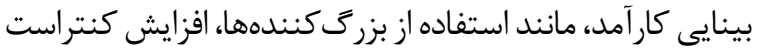

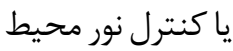

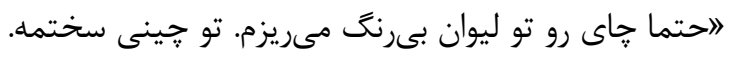

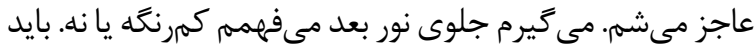

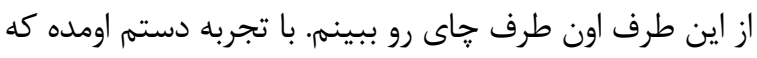
جقدر معلوم باشه خوبه." إن طرفي

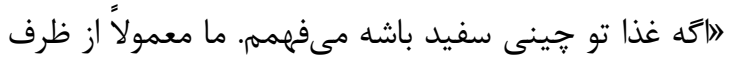

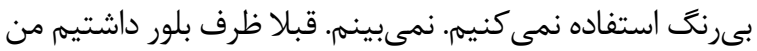

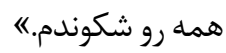

\section{بحث}

هدف از اين مطالعه، به دست آوردن راهكارهاى منحصربهافردى

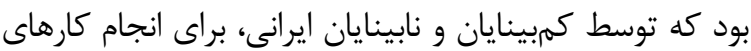
روزمره به كار كرفته مىشد.

افراد داراى كاهش بينايى، در انجام امورى كه افراد عادى، با باديا

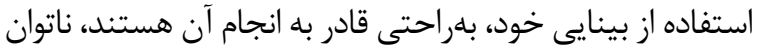

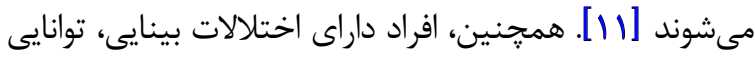

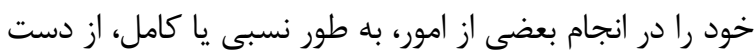

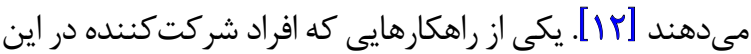

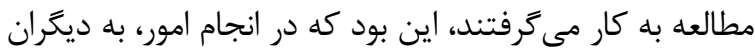

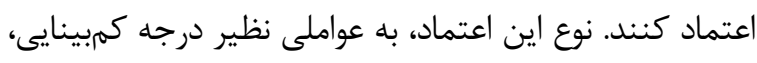

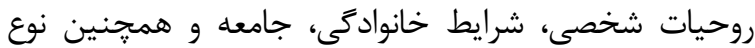

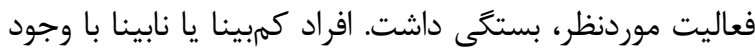

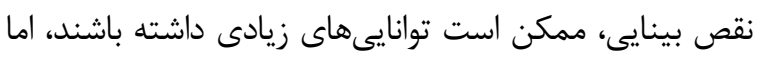

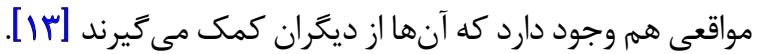
نابينايان، توانايى قابل توجهى در استفاده از ساير حواس، براى

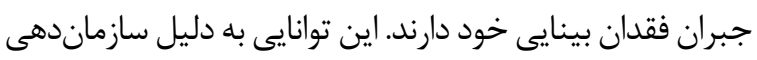

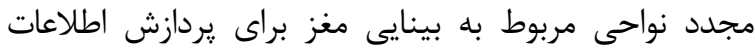

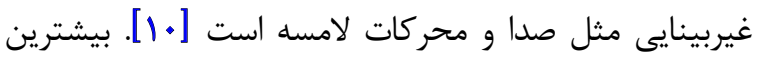

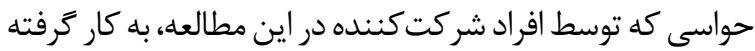

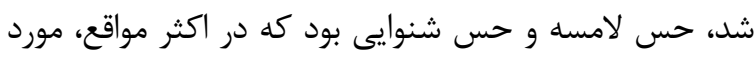

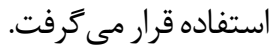

ديكر استراتثى به كار كرفتهشده، استفاده از تكنولوزى بود.

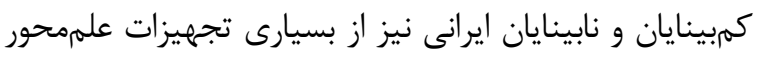

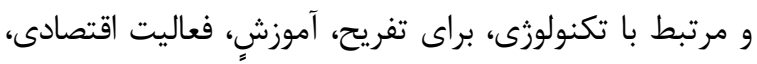

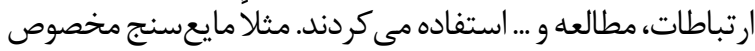

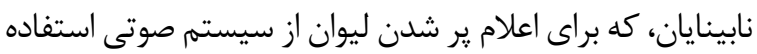

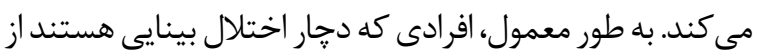

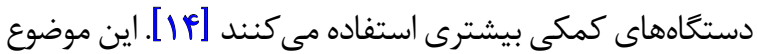

$$
\text { در ايران هم صدق مىكرد. }
$$

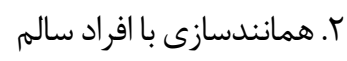

الموقع مسواك زدن، موقعى كه موهام رو شونه مى كنم يا قراره

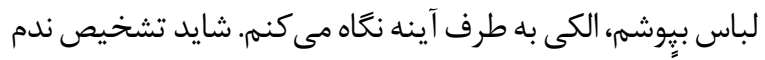

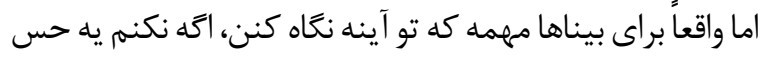
بدى بهشون دست مى خده، به خاطر اونا اين كار رو مى كنمه و اينكه

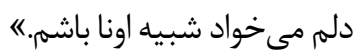
r. افزايش اعتمادبهنفس و خودباورى خايد بزركترين تغييرى كه من توى خودم دادم رفتارمه. به رونه

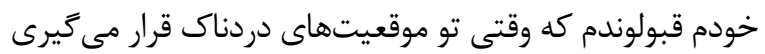

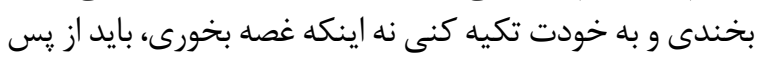

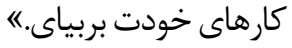
بل كارتيرى هوش و حافظظه بهكاركيرى هوش و حافظه، استراتزى ديخرى بود كه شركت كنند انان، به كار مى ترفتند.

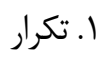
"يه آدرس بخوام برم، كلاً بايد جند بار برم ديخه ياد مى گيرم."

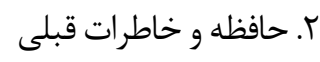
"اموسيقى براى نابينا خيلى سخت هر از يك بيناست. جون بينا

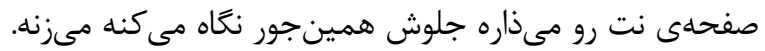

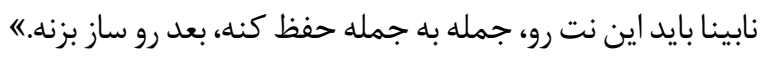

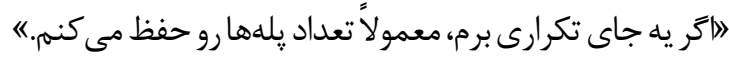
r. برقرارى ارتباط منطقى با امور

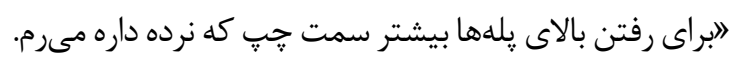

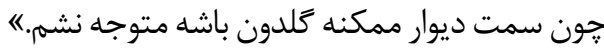
؟ا. در نظر گرفتن زمان مورد نياز براى انجام دادن امور

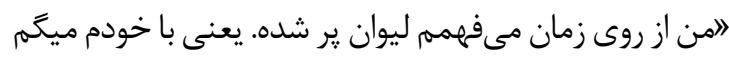

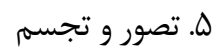

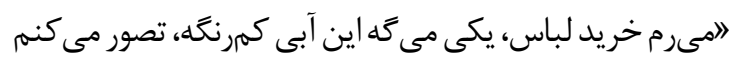

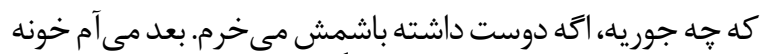

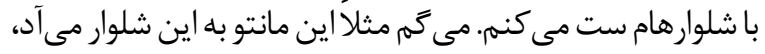

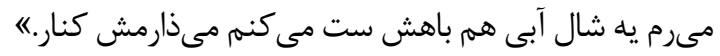
هراقبث از بيئايي كارآمد تعداد كمى از شركت كنندكان، علىرغم كاهش شديد بينايى، همجنان از بينايى خود استفاده مى كردند. 
عادى انجام مى دادند. مانند نحًاه كردن در آينه هنَام شانه زدن

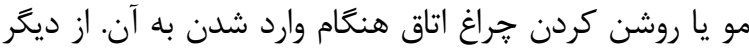

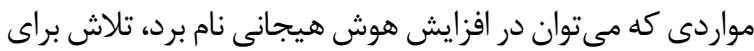

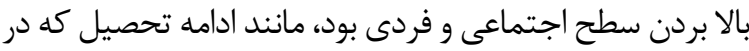
تعدادى از شركت كنند كان ديده مى شئد.

از نظر بسيارى از روانشناسان، هوش مجموعه استعدادهايى

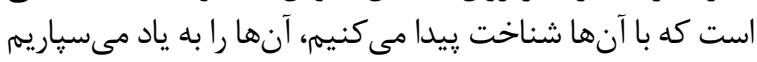

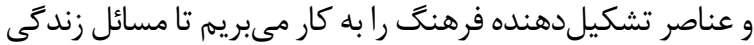

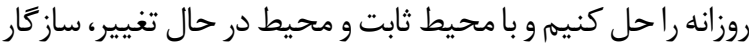

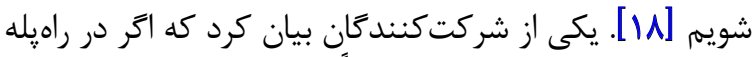

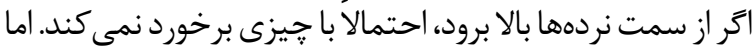

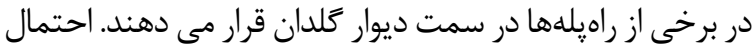

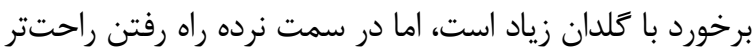

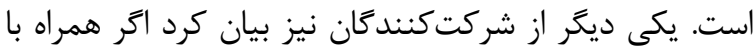

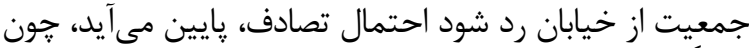

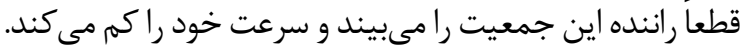
تصور، درعمل يك نوع خلق كردن است، مانند داستانى كه

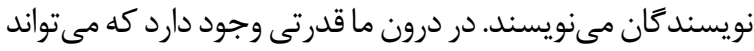

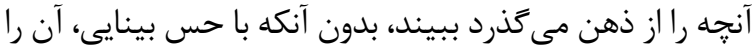

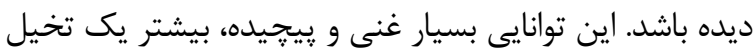

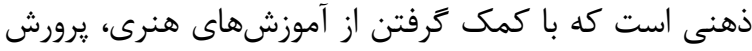

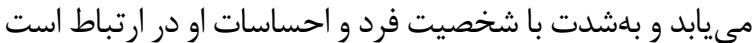

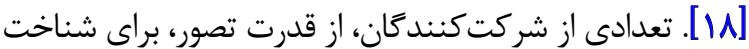

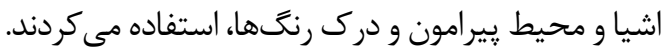

بينايى كارآمد، زمانى تعريف مىشود كه به به واسطه كاهش

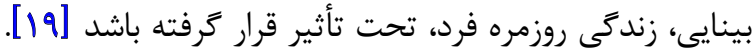

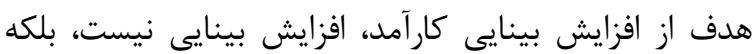

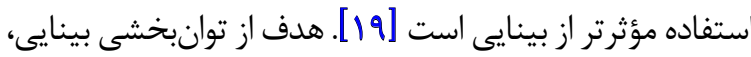

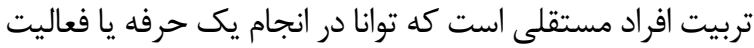

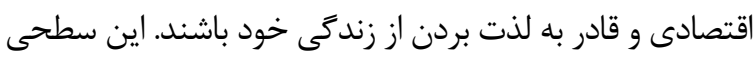

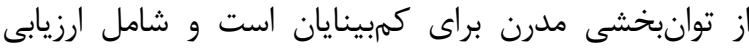

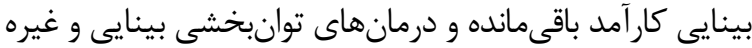

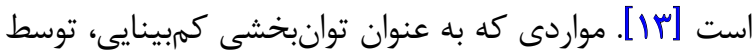

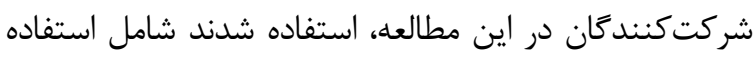

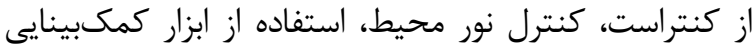

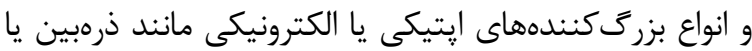

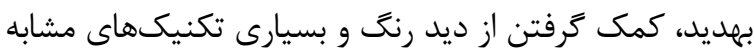
بود. در بين شركت كنند

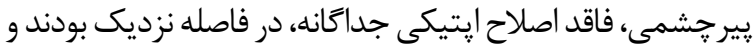

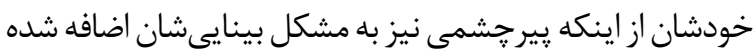

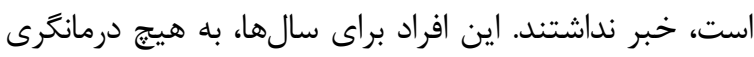

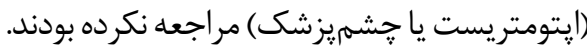

مهرمترين روش تحرك و جهتيابى، استفاده از عصاى سفيد

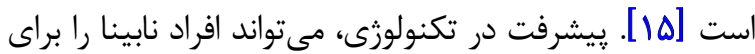

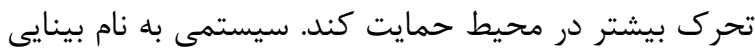

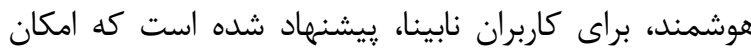

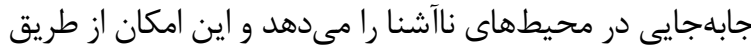

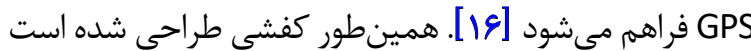

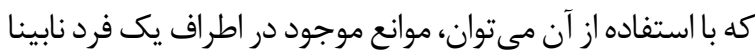

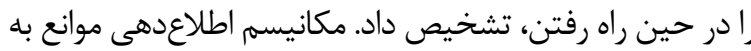

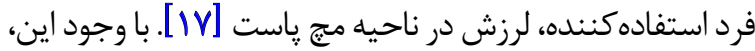
كمبينايان و نابينايان ايرانى، از عصاهاى قديمى فيمى استفاده مى كردند. تعدادى از شركت كنند

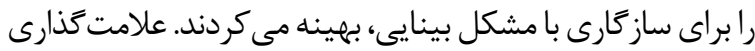

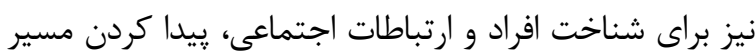

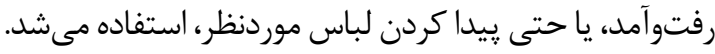

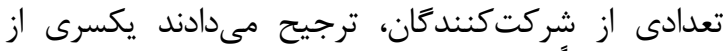

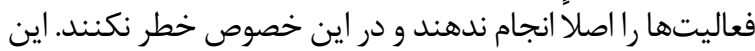

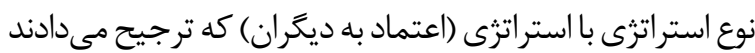

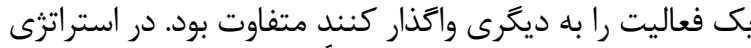

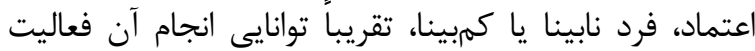

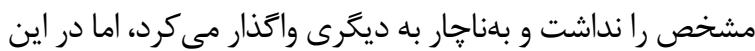

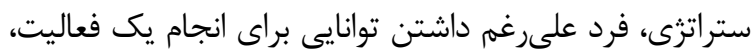

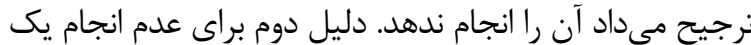

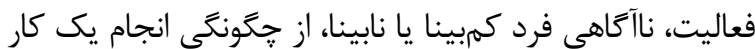

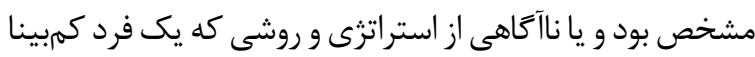
يا نابيناى مشابه، براى انجام آن كار، به كار مى رَّرت يكى ديخر از استراترىها، بهكار كيرى هوش هيجانى بود. هوش

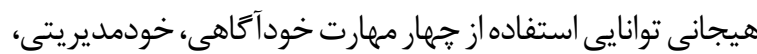

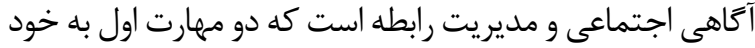

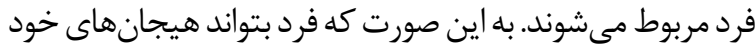

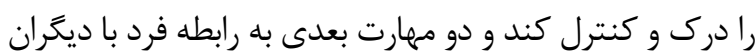

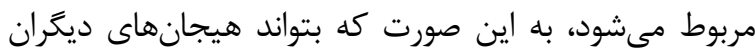

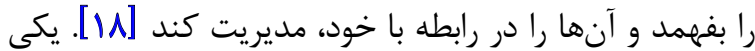

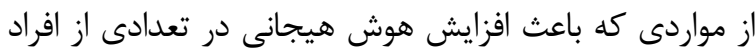

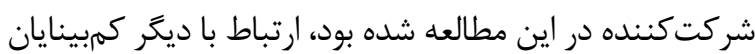

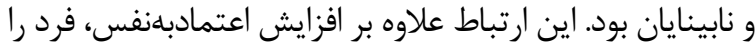

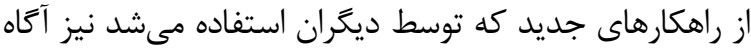

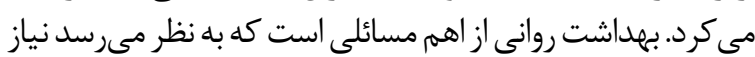

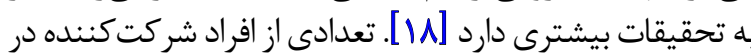

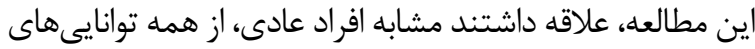

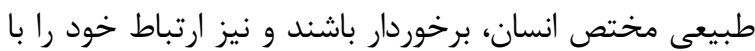

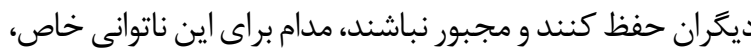

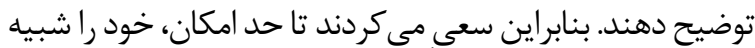
ديخران نشان دهند، و دقيقاً همان كارى رئ را انجام دهند كاند كه افراد 


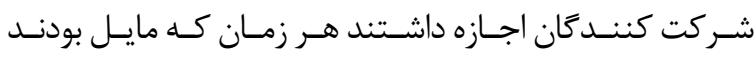

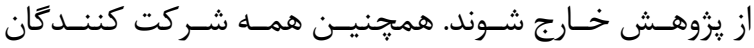

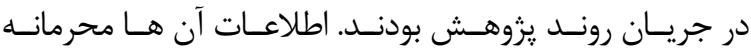

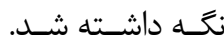

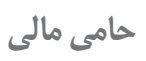

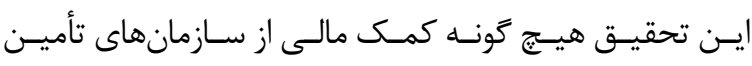

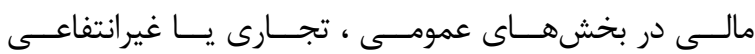

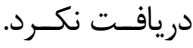

$$
\text { مشار كت نويسندًان }
$$

تمــام نويســند

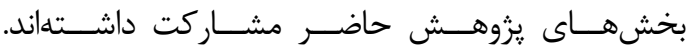

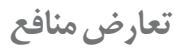

بنابر اظهار نويسندكان اين مقاله تعارض منافع ندارد.

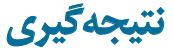

كمبينايان و نابينايان ايرانى از استراترىهاى منحصربهفردي

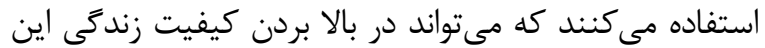

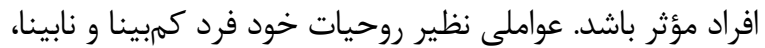

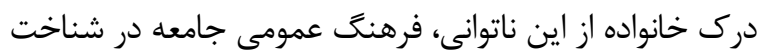

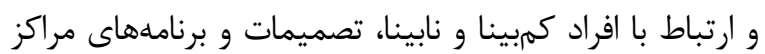

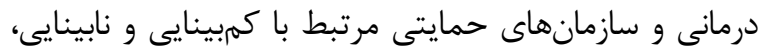

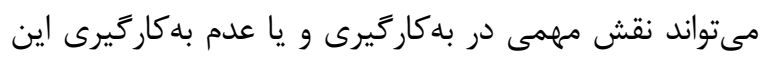

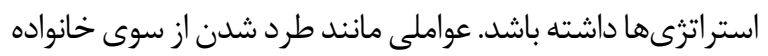

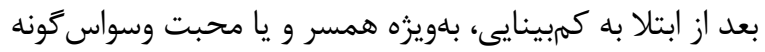

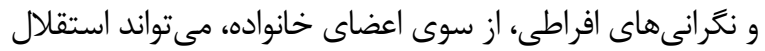

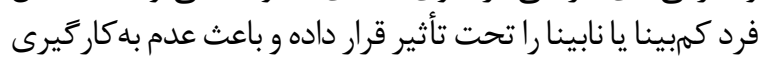

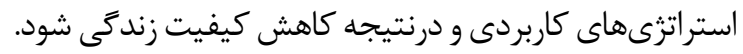
با توجه به اينكه مطالعه حاضر به صورت كيفى انجام شد،

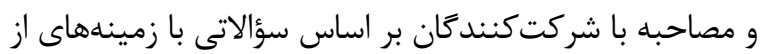

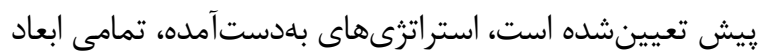

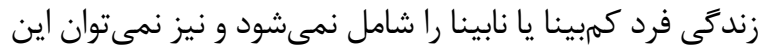

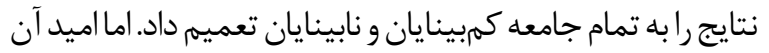

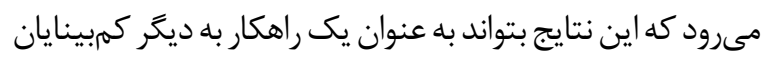

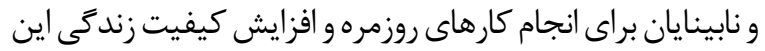
قشر از جامعه، يُيشنهاد شود.

در يزوهش حاضر، علت كمبينايى و نابينايى، در انتخاب

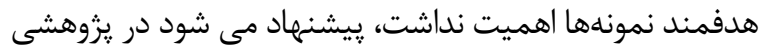

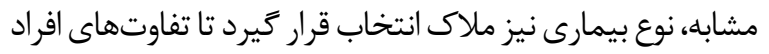

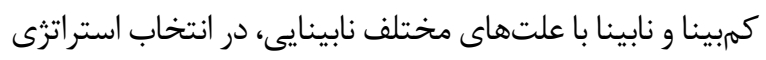

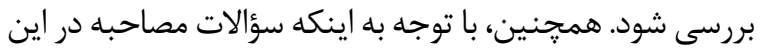

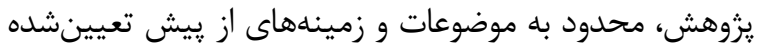

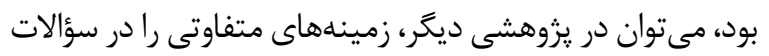

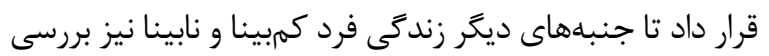

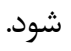
اين يزوهش حاصل نتايج يايانامه كارشناسى ارشد است.

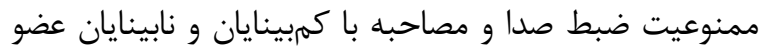

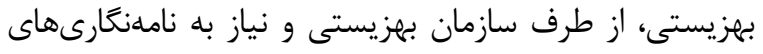

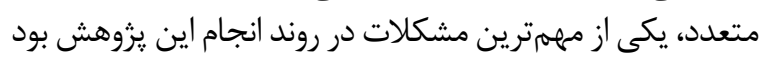

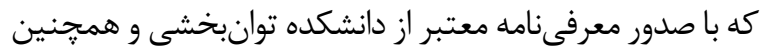

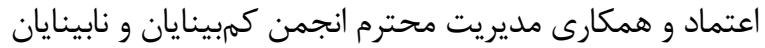
خزانه، تا حدودى مشكلات مرتفع شد.

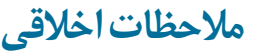
يبروى از اصول اخلاق يزوهش اصـول اخلاقـى تمامــاً در ايـن مقالـهـ رعايـت شــده اســت. 


\section{Referencs}

[1] Congdon NG, Friedman DS, Lietman T. Important causes of visual impairment in the world today. JAMA. 2003; 290(15):2057-60 [DOI:10.1001/jama.290.15.2057]

[2] The Eye Diseases Prevalence Research Group. Causes and prevalence of visual impairment among adults in the United States. Archives of Ophthalmology. 2004; 122(4):477-85. [DOI:10.1001/ archopht.122.4.477]

[3] Rai P, Rohatgi J, Dhaliwal U. Coping strategy in persons with low vision or blindness - an exploratory study. Indian Journal of Ophthalmology. 2019; 67(5):669-76. [DOI:10.4103/ijo.IJO_1655_18]

[4] Anil K, Garip G. Coping strategies, vision-related quality of life, and emotional health in managing retinitis pigmentosa: A survey study. BMC Ophthalmology. 2018; 18:21. [DOI:10.1186/s12886018-0689-2]

[5] Sturrock BA, Xie J, Holloway EE, Lamoureux EL, Keeffe JE, Fenwick EK, et al. The influence of coping on vision-related quality of life in patients with low vision: A prospective longitudinal study. Investigative Ophthalmology \& Visual Science. 2015; 56(4):2416-22. [DOI:10.1167/iovs.14-16223]

[6] Brouwer DM, Sadlo G, Winding K, Hanneman MIG. Limitations in mobility: Experiences of visually impaired older people. British Journal of Occupational Therapy. 2008; 71(10):414-21. [DO:10.1177/030802260807101003]

[7] Stevelink SAM, Fear NT. Psychosocial impact of visual impairment and coping strategies in female ex-Service personnel. BMJ Military Health. 2016; 162(2):129-33. [DOI:10.1136/ jramc-2015-000518]

[8] Boerner K, Wang SW. Goals with limited vision: A qualitative study of coping with vision-related goal interference in midlife. Clinical Rehabilitation. 2012; 26(1):81-93. [DOI:10.1177/0269215511407219]

[9] Jackson WT, Taylor RE, Palmatier AD, Elliott TR, Elliott JL. Negotiating the reality of visual impairment: Hope, coping, and functional ability. Journal of Clinical Psychology in Medical Settings. 1998; 5(2):173-85. [DOI:10.1023/A:1026259115029]

[10] Askari S, Shafaroudi N, Kamali M, Khalaf Beigi M. [Environment and blindness: The experiences of adults with acquired blindness in performing the activities of daily living (Persian)]. Middle Eastern Journal of Disability Studies. 2011; 1(1):29-41. http://idisabilstud.org/article-1-315-fa.html

[11] Richman J, Lorenzana LL, Lankaranian D, Dugar J, Mayer JR, Wizov SS, et al. Relationships in glaucoma patients between standard vision tests, quality of life, and ability to perform daily activities. Ophthalmic Epidemiology. 2010; 17(3):144-51. [DOI:10.3109/09286581003734878

[12] Osaba M, Doro J, Liberal M, Lagunas J, Kuo IC, Reviglio VE. Relationship between legal blindness and depression. Medical Hypothesis, Discovery \& Innovation Ophthalmology Journal. 2019; 8(4):306-11. [PMID] [PMCID]

[13] Stevens S. Assisting the blind and visually impaired: Guidelines for eye health workers and other helpers. Community Eye Health. 2003; 16(45):7-9. [PMID] [PMCID]
[14] Dahlin-Ivanoff S, Sonn U. Use of assistive devices in daily activities among 85 -year-olds living at home focusing especially on the visually impaired. Disability and Rehabilitation. 2004; 26(24):1423-30. [DOI:10.1080/096382804000000906]

[15] Beh-Pajooh A, Taherian M, Shekar Roo M. [The relationship between mobility and orientation using white cane and mental health and personality traits among individuals with visual impairment (Persian)]. Journal of Exceptional Children. 2018; 18(2):99-112. http://joec.ir/article-1-658-en.html

[16] Faria J, Lopes S, Fernandes H, Martins P, Barroso J. Electronic white cane for blind people navigation assistance. Paper presented at: 2010 World Automation Congress. 2010 19-23 September 2010, Kobe, Japan. https://ieeexplore.ieee.org/document/5665289

[17] Goudarzi R, Jamshidi N. [A new intelligent shoe for blind people using ultra sound waves for obstacle detection base on human gait cycle (a replacement for white cane) (Persian)]. Iranian Journal of War and Public Health. 2013; 5(2):1-9. http://ijwph. ir/article-1-245-en.htm

[18] Ganji H. [General psychology (Persian)]. Tehran: Savalan; 2013. http://opac.nlai.ir/opac-prod/bibliographic/3459378

[19] Riazi A. [Low vision children (Persian)]. Kashan: Mohtasham; 2016. http://opac.nlai.ir/opac-prod/bibliographic/4837886 
This Page Intentionally Left Blank 